\title{
Expression profiling analysis of autophagy-related genes in perineural invasion of cutaneous squamous cell carcinoma
}

\author{
LI-QIANG ZHENG ${ }^{1,2 *}$, SHAN-YI LI ${ }^{*}$ and CHENG-XIN LI ${ }^{1}$ \\ ${ }^{1}$ Department of Dermatology, Chinese PLA General Hospital, Beijing 100853; \\ ${ }^{2}$ Department of Dermatology, The 251st Hospital of Chinese PLA, Zhangjiakou, Hebei 075000, P.R. China
}

Received July 19, 2017; Accepted December 19, 2017

DOI: $10.3892 / \mathrm{ol} .2018 .7971$

\begin{abstract}
The aim of the present study was to identify the potential autophagy-related genes and to explore the underlying molecular mechanisms involved in cutaneous squamous cell carcinoma of head and neck (cSCCHN) by bioinformatics analysis. The Gene Expression Omnibus (GEO) series GSE86544 was downloaded from the GEO database. The primary data was generated from $\mathrm{cSCCHN}$ with clinical perineural invasion (PNI) and cSCCHN without PNI, and was further analyzed in order to identify differentially expressed genes (DEGs). The results revealed 239 autophagy-related DEGs. Gene Ontology (GO) and Kyoto Encyclopedia of Genes and Genomes (KEGG) enrichment analyses were performed and intersected to investigate the predicted functions of the key DEGs, including hypoxia-inducible factor $1 \alpha$ (HIF1A), mitogen-activated protein kinase 8 (MAPK8), mammalian target of rapamycin (mTOR) and B-cell lymphoma 2 like 1 (BCL2L1). Up and downregulated genes shared one pathway, namely 'pathways in cancer'. Next, the protein-protein interaction (PPI) network of the autophagy-related DEGs was constructed using Cytoscape 3.30 software. HIF1A, MAPK8, mTOR and BCL2L1 were key nodes in the PPI network. Additionally, RAB23 gene expression was positively correlated with HIF1A, MAPK8 and ADP ribosylation factor GTPase activating protein 1 (ARFGAP1), but negatively correlated with mTOR and BCL2L1. The present results suggested that the genes HIF1A, MAPK8, mTOR, BCL2L1 and RAB23 may be associated with and serve as potential therapeutic targets in cSCCHN with clinical PNI.
\end{abstract}

Correspondence to: Dr Cheng-Xin Li, Department of Dermatology, Chinese PLA General Hospital, 28 Fuxing Road, Beijing 100853, P.R. China

E-mail: chengxinderm@163.com

*Contributed equally

Key words: autophagy, perineural invasion, cutaneous squamous cell carcinoma, bioinformatics

\section{Introduction}

Cutaneous squamous cell carcinoma (cSCC) is the second most common keratinocyte cancer and accounts for $\sim 30 \%$ of all non-melanoma skin malignant tumors $(1,2)$. The 5-year recurrence rate and metastasis of primary lesions is 8 and 5\%, respectively. The fatality rate of cSCC metastasis is $\sim 40 \%$ (2). In general, long-term exposure to ultraviolet light is the strongest risk factor, so the most frequent sites of disease are the sun-exposed regions of head and neck (3). Unless high-risk features are present, the overwhelming majority of $\mathrm{cSCC}$ is curable with operation, radiotherapy and/or chemotherapy (4). However, once invasion and metastasis have occurred, the prognosis is poor.

Increasing evidence has demonstrated that tumorigenesis, progression, invasion and metastasis of SCC involve several signal transduction pathways and molecules, including Notch, Integrin, tumor protein p53 (p53), epidermal growth factor receptor (EGFR) (5). Recently, researchers have demonstrated that autophagy might play a pivotal role in the pathogenesis of cSCC $(6,7)$. Autophagy (term here used generally for macroautophagy) is a homeostatic mechanism under starvation or stressful conditions, resulting in the degradation of aggregated proteins and damaged organelles, and subsequently buffering metabolic stress by recycling intracellular components $(8,9)$. The dysregulation of autophagy is closely related with various pathological diseases, including cardiomyopathy, muscular diseases, neurodegenerative disorders, infection and cancer (10).

In cancer cells, autophagy demonstrates both tumor-promoting and tumor-repressing properties, generally depending on the cell type and context. During the initiation of cancer, autophagy may mediate elimination of altered cytosolic products, such as long-lived proteins or damaged organelles, preventing cells from further DNA damage, genomic instability and tumorigenesis $(11,12)$. Once the cancer has formed, autophagy contributes to tumor development by allowing tumor cells to survive under starvation or hypoxia conditions (13). Autophagy has a 'double swords' role in cSCC, but there is no definite time threshold for when autophagy may promote SCC tumorigenesis or suppression, because the initial time of pathogenesis is usually earlier than that of the clinical diagnosis. Therefore, the relationship between autophagy and SCC pathogenesis and progression is complex. Previous studies 
have, however, indicated that autophagy-specific markers [high expression of Beclin 1 and/or microtubule-associated protein 1 light chain 3 (LC3), and lower level of p62/sequestosome-1] may serve a key role in controlling the tumorigenesis, progression and lymph-node metastasis of cSCC and may be prognostic predictors of clinical outcome $(6,7,14)$.

Furthermore, in human squamous cell carcinoma of head and neck (SCCHN), autophagy-related signaling pathways, such as phosphoinositide 3-kinase (PI3K)/AKT/mammalian target of rapamycin (mTOR) or extracellular signal-regulated kinase (ERK) are activated in some cases. Autophagy also alleviates apoptotic cell death in SCCHN. Concomitant inhibition of AKT and autophagy enhances efficient cisplatin-induced apoptosis in metastatic cSCC (15-17). During invasion of cSCC, epithelial-mesenchymal transition is paralleled by AKT activation and AKT may serve as a treatment target to prohibit dissemination of cSCC (18). However, contrary evidence has suggested that activation of autophagic pathways is associated with growth inhibition and senescence in metastatic cSCC (19).

Notably, perineural invasion (PNI) is usually defined as tumor cells invading into the perineural space, well-recognized as one of the high-risk factors of cSCC. The detection of PNI in cSCC indicates an aggressive tumor and predicts a worse outcome, with higher rates of local recurrence, metastases and poor survival (20). Clinical PNI displays features of spread along central nerves, based on clinical, radiological or histological evidence. Compared with incidental PNI, clinical PNI is associated with a worse prognosis (20) and requires a more aggressive treatment approach. Furthermore, the 5-year overall survival rate for clinical PNI in SCC ranges from $56-64 \%(21,22)$.

Although accumulating studies have explored the role of autophagy-related genes in prognosis of this disease, most of the previous study has been limited to analyzing one or two genes in cell lines, tissue or animal models. At present, gene expression analysis provides an efficient method for processing mass data. For example, the finding of similar differentially expressed genes (DEGs) in actinic keratosis (AK) and SCC by Padilla et al (23) and Ra et al (24) confirmed that AK is a precursor lesion of SCC and signified that both are closely related genetically. In invasive SCC, gene analysis results suggested that IL-24 contributed to SCC invasion through enhancing focal expression of matrix metalloproteinase (MMP) 7 (25). In addition, Ephrin type-B receptor 2 (EphB2) knockdown downregulated the gene expression of MMP1 and MMP13, which are associated with biological functions such as cell viability, migration and invasion of tumor cells (26). Warren et al (22) reported that alterations in the p53 pathway may be important in cSCC of head and neck (cSCCHN) with clinical PNI, but not in cSCCHN without PNI (22). However, few analysis results of gene expression signatures focusing on autophagy in cSCC invasion have been documented. In addition, previous study from our research group has demonstrated that RAB23 knockdown repressed cell invasion, while RAB23 overexpression promoted cell invasion, depending on the GTP-bound form of RAB23 (27). Therefore, whether RAB23 promotes cSCC invasion may be directly associated with the autophagy process. However, the association among RAB23 expression, autophagy and tumor cells PNI in cSCCHN has not been defined.
In the present study, expression differences of autophagy-related genes between cSCCHN and cSCCHN with clinical PNI were explored. The original data series GSE86544 was downloaded from Gene Expression Omnibus (GEO; http://www.ncbi.nlm.nih.gov/geo/). Then autophagy-related gene expression profiles were screened within the DEGs between cSCCHN and cSCCHN with clinical PNI. In addition, a RAB23-related gene regulation network (http://autophagy-regulation.org/) was obtained. To explore their biological functions and pathways, the autophagy-related DEGs were further examined by Gene Ontology (GO) and pathway enrichment analysis. Next, protein-protein interaction (PPI) networks and sub-networks were constructed and analyzed. Finally, the relationship among RAB23 expression, autophagy-related key DEGs and tumor cells PNI was explored in cSCCHN. Collectively, the present results demonstrated that a close correlation exists among RAB23, autophagy and cSCCHN with clinical PNI and that targeting these autophagy-related genes may be a promising therapeutic strategy for cSCC.

\section{Materials and methods}

Microarray data. The microarray expression profile dataset GSE86544, submitted by Warren et al (22) and based on the GEO Platform10588 Illumina HumanHT-12 V4.0 expression beadchip (Illumina, Inc., San Diego, CA, USA), was downloaded from the GEO database (http://www.ncbi.nlm. nih.gov/geo/). The dataset contained 24 samples, including 9 cSCCHN, 7 cSCCHN with incidental PNI and 8 cSCCHN with clinical PNI. In the present study, the cSCCHN with clinical PNI or without PNI samples were analyzed by bioinformatics. The dataset was downloaded from the GEO database on February 2, 2017.

Data preprocessing and analysis of DEGs. The original microarray data were transformed into expression measures. Background correction, quartile data normalization and probe synopsis were performed with GEO2R online, using the Robust Multi-array Average (RMA) algorithm in the Raffy package (https://www.bioconductor. org/packages/release/bioc/html/affy.html). To obtain the P-value, multiple testing corrections were applied with the Benjamini-Hochberg method. Only those genes exhibiting $\log 2$ fold change $(\mathrm{FC})>1.5$ and adjusted $\mathrm{P}<0.05$ were considered to be DEGs. When multiple probes matched to the same gene, the average expression value of probes was calculated. Nonspecific probes were filtered.

Autophagy-related genes (https://www.ncbi.nlm. nih.gov/gene) were downloaded by using the key words 'autophagy' and 'Homo sapiens'. The 907 autophagy-related genes were obtained as a symbol gene list, and was compared with the list of DEGs. The comparison resulted in a list of 239 autophagy-related DEGs. The RAB23-related gene regulation network (http://autophagy-regulation.org/) (28) was also searched, which is compatible with Cytoscape software.

GO and Kyoto Encyclopedia of Genes and Genomes (KEGG) enrichment analysis. The Database for Annotation, Visualization and Integrated Discovery (DAVID 6.8; 


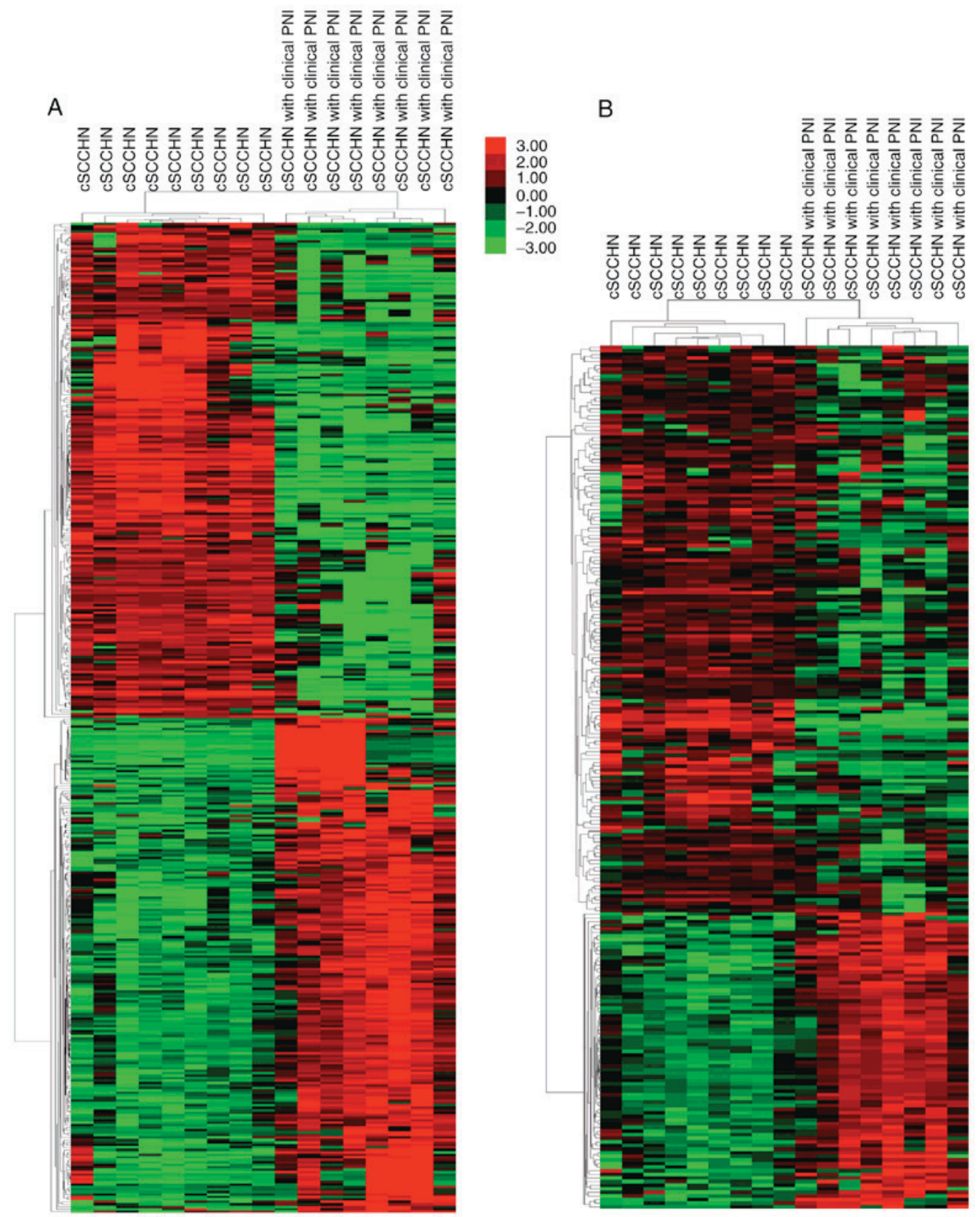

Figure 1. DEG expression illustrated in heat maps. (A) Heat map of DEGs in cSCCHN with clinical PNI compared with cSCCHN without PNI (top 200 upregulated and downregulated genes). (B) Hierarchical clustering of the expression values of autophagy-related DEGs. DEG, differentially expressed gene; cSCCHN, cutaneous squamous cell carcinoma of head and neck; PNI, perineural invasion.

https://david.ncifcrf.gov/) consists of a set of functional annotation tools, which have been developed for linking functional terms with lists of genes by clustering algorithms (29). To analyze the identified DEGs at the functional level, GO enrichment analysis was performed to annotate genes or gene products and identify characteristic biological properties for genome or transcriptome data using the DAVID online tool. Additionally, the KEGG pathway database was used for systematic analysis of gene functions and related pathways. $\mathrm{P}<0.05$ was considered to indicate a statistically significant difference.

Hierarchical clustering of DEGs and data visualization. The series matrix file for GSE86544 was obtained from GEO DataSets (downloaded on February 2nd, 2017). The Pearson's correlation coefficient was performed using Cluster 3.0 with average linkage in order to achieve the hierarchical heatmap (30). Analysis results were visualized employing the Java TreeView 1.1.6 version (31).

PPI network construction. The Search Tool for the Retrieval of Interacting Genes (STRING; version 10.0) covers $5,214,234$ proteins from 1,133 organisms (32). In the present study, to evaluate the interactive relationships among DEGs, the 239 DEGs were mapped to the STRING database, and only experimentally validated interactions (combined score $>0.4$ ) were selected as significant.

PPI networks were constructed using the Cytoscape 3.3.0 software (http://www.cytoscape.org/). The degree of connectivity is evident by the number of edges linked to a given 

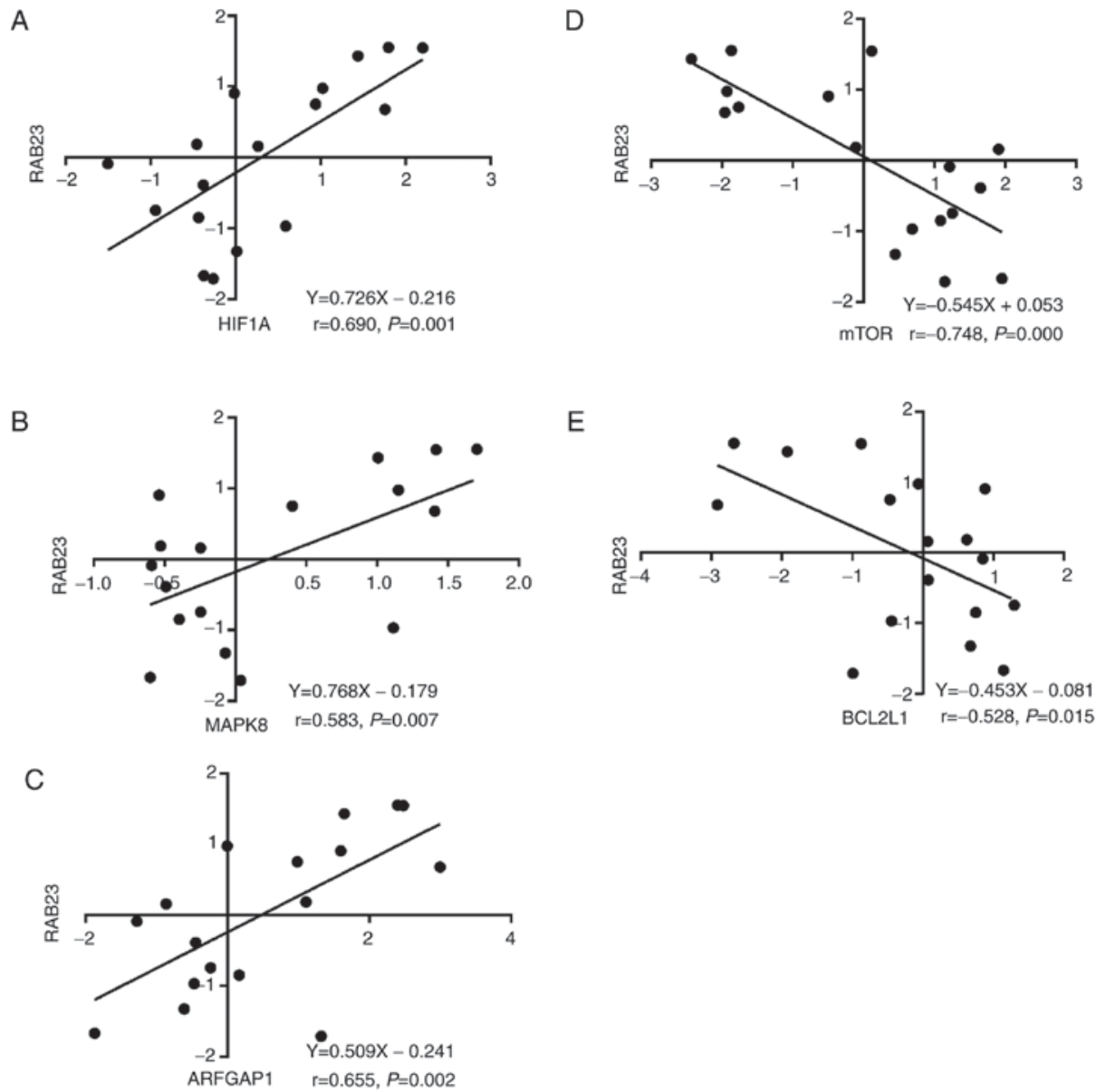

Figure 2. RAB23 gene expression was positively correlated with (A) HIF1A, (B) MAPK8 and (C) ARFGAP1 expression, but negatively correlated with (D) mTOR and (E) BCL2L1 expression. HIF, hypoxia-inducible factor; MAPK8, mitogen-activated protein kinase 8; ARFGAP1, ADP ribosylation factor GTPase activating protein 1; mTOR, mammalian target of rapamycin; BCL2L1, B-cell lymphoma 2 like 1.

node. The nodes with a high degree are regarded as the key genes that possess vital biological functions. Then, the degree of connectivity was analyzed to obtain the key proteins in the PPI network. The plug-in Molecular Complex Detection (MCODE) was used to screen the modules of the PPI network (MCODE scores $>30$ and number of nodes $>4$ ) in Cytoscape.

Statistical analysis. The relationship between RAB23 and the key genes was analyzed using SPSS 17.0 software (SPSS, Inc., Chicago, IL, USA). P $<0.05$ was considered to indicate a statistically significant difference.

\section{Results}

Identification of DEGs. Following data processing, a total of 2,858 DEGs were identified in cSCCHN with clinical PNI, compared with cSCCHN samples without PNI involvement. These DEGs contained 921 upregulated and 1,937 downregulated genes, and the top 200 up and downregulated genes were clustered (Fig. 1A). In comparison to DEGs, 239 autophagy-related genes were screened and were also clustered employing the Series Matrix Files (Fig. 1B).

GO and pathway enrichment analysis. The 239 autophagy-related DEGs were uploaded to the online DAVID tool in order to identify over-represented GO categories and
KEGG pathways. The results from GO analysis demonstrated that upregulated DEGs mainly focused in biological processes (BP), including tumor necrosis factor-mediated signaling pathway, autophagy, positive regulation of MAP kinase activity, and ERK1 and ERK2 cascade. The downregulated DEGs were significantly enriched in autophagy, signal transduction, macroautophagy and positive regulation of gene expression (Table I).

By KEGG analysis, the upregulated DEGs of autophagy mainly focused on cytokine-cytokine receptor interaction, pathways in cancer, ErbB signaling pathway, hypoxia inducible factor (HIF)-1 signaling pathway and transcriptional misregulation in cancer. By contrast, the downregulated DEGs of autophagy distributed in insulin resistance, pathways in cancer, forkhead box $\mathrm{O}$ (FoxO) signaling pathway and insulin signaling pathway (Table II).

Finally, the intersection of genes from GO and KEGG pathway analysis was obtained, including as the upregulated genes HIF1A and mitogen-activated protein kinase 8 (MAPK8), and the downregulated genes mammalian target of rapamycin (mTOR) and B-cell lymphoma 2 like 1 (BCL2L1). These genes were considered to be key genes in cSCCHN with clinical PIN. In addition, as RAB23 gene expression was positively correlated with HIF1A ( $\mathrm{P}=0.001, \mathrm{r}=0.690$; Fig. 2A), MAPK8 ( $\mathrm{P}=0.007, \mathrm{r}=0.583$; Fig. $2 \mathrm{~B})$ and ADP ribosylation factor GTPase activating protein 1 (ARFGAP1; $\mathrm{P}=0.000$, 


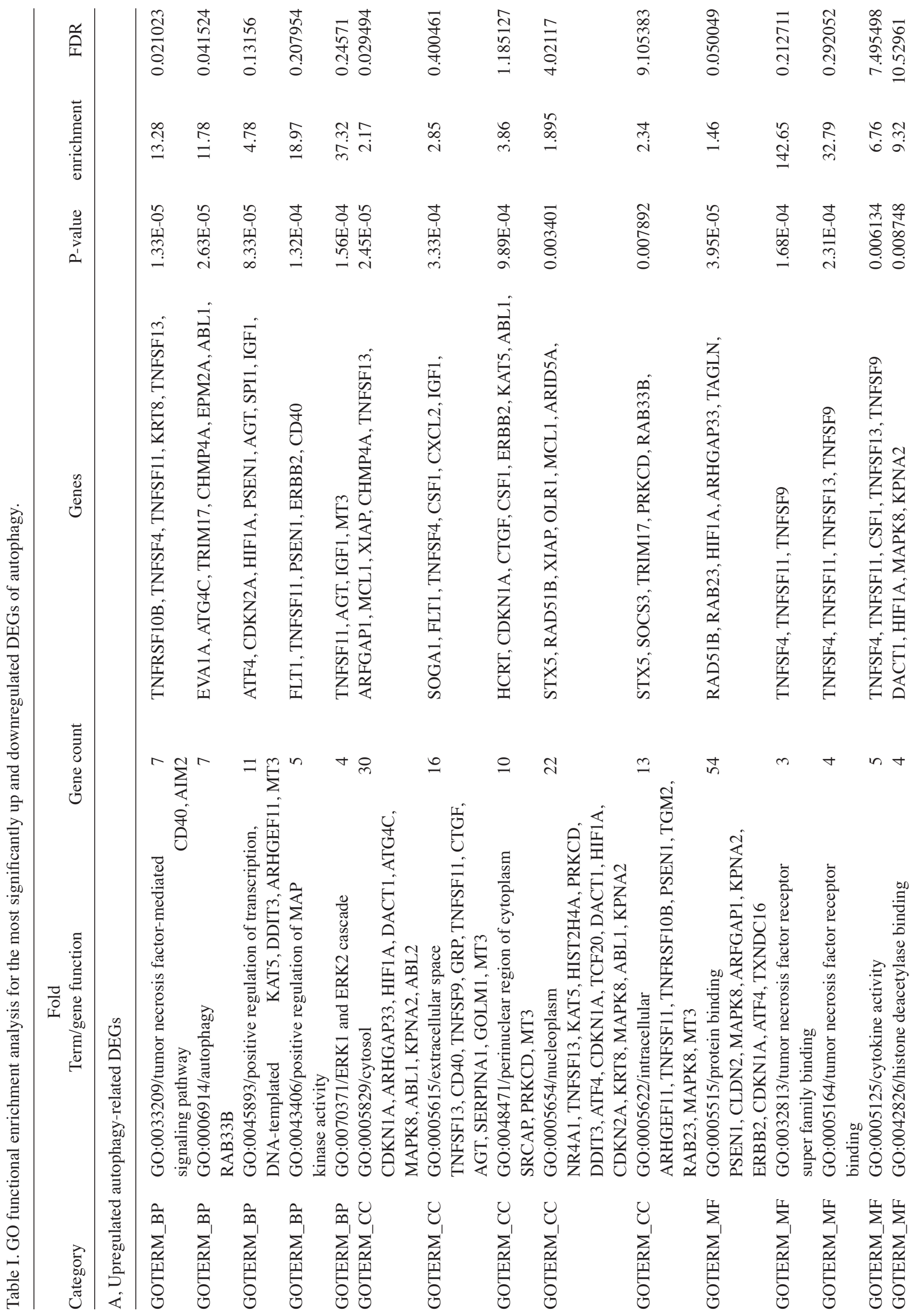




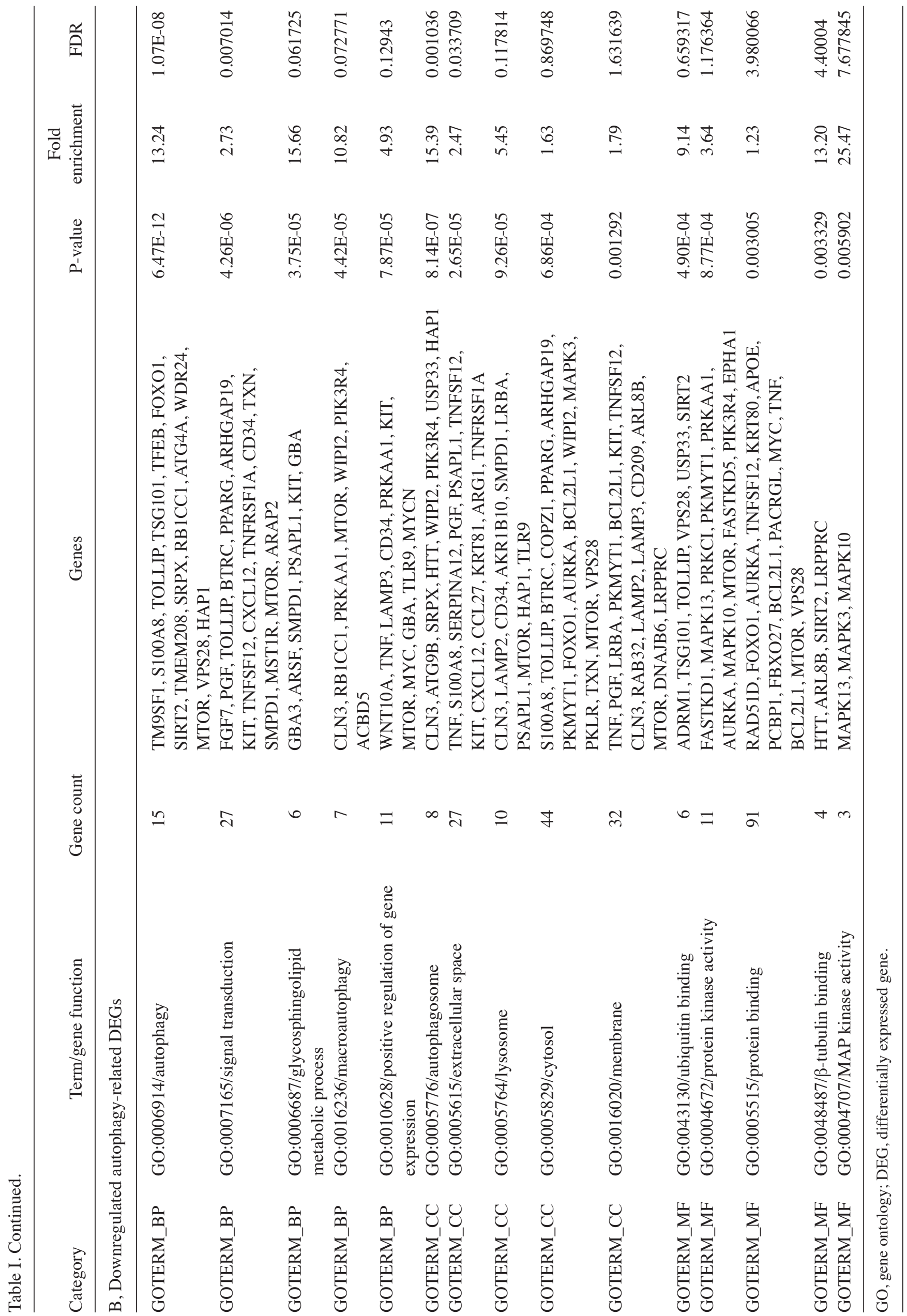




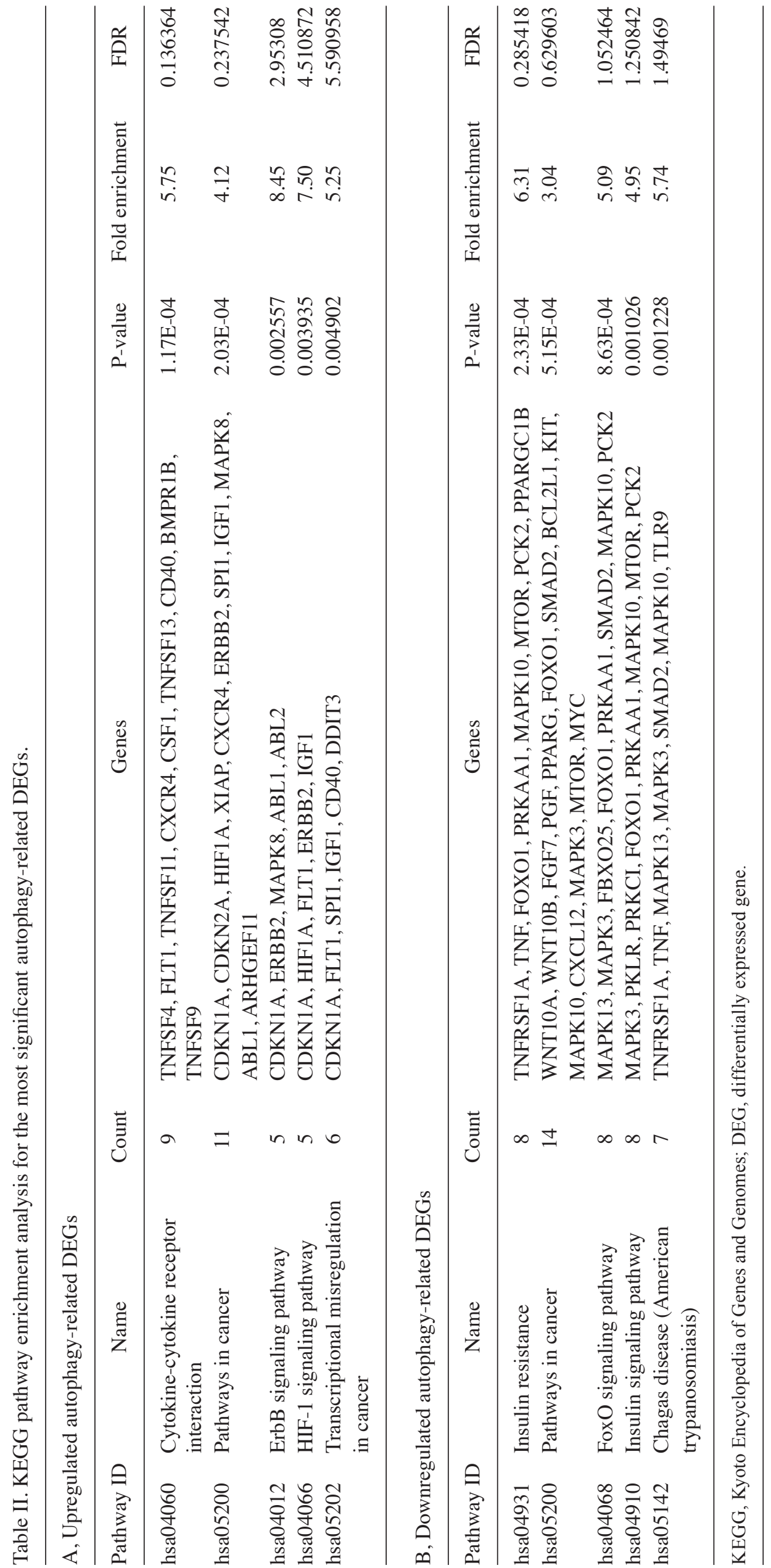




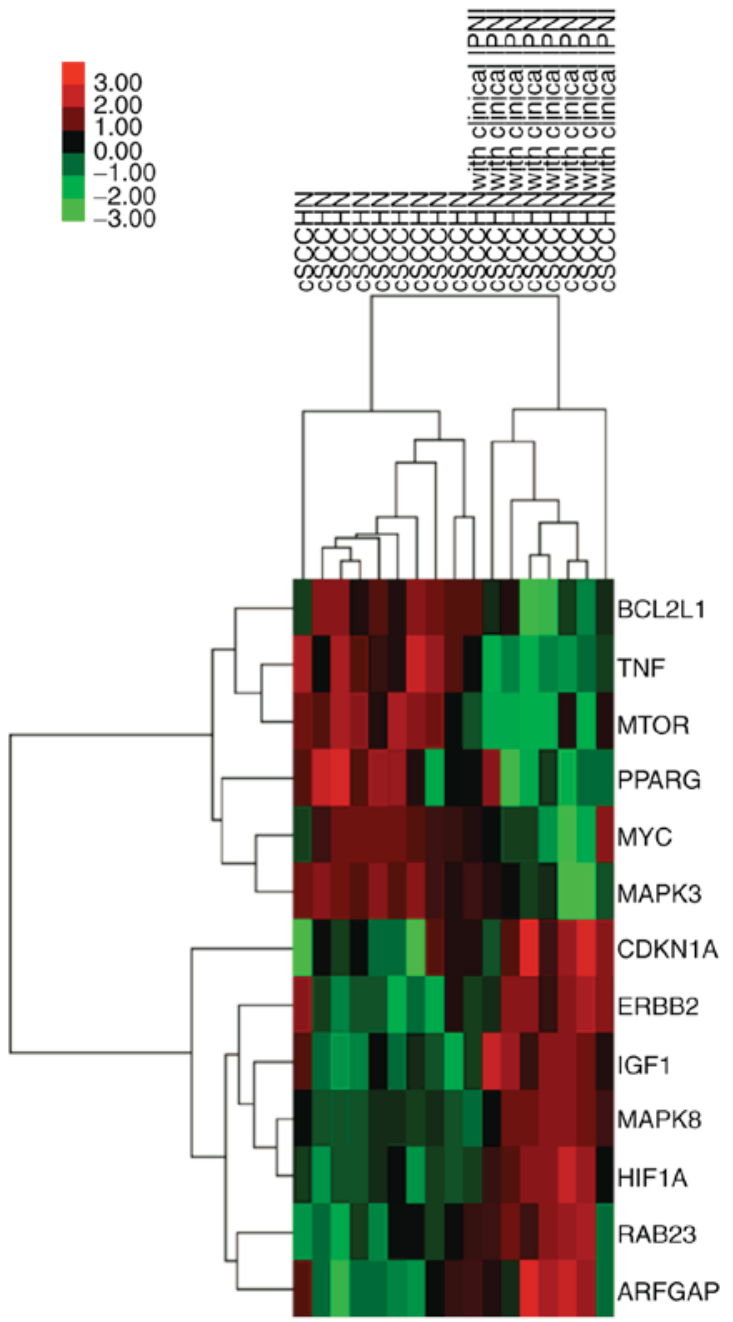

Figure 3. Heatmap of RAB23 expression and its related genes indicates that RAB23 is positively associated with HIF1A, MAPK8 and ARFGAP1 expression, and negatively associated with mTOR and BCL2L1 expression. HIF, hypoxia-inducible factor; MAPK8, mitogen-activated protein kinase 8; ARFGAP1, ADP ribosylation factor GTPase activating protein 1; mTOR, mammalian target of rapamycin; BCL2L1, B-cell lymphoma 2 like 1; cSCCHN, cutaneous squamous cell carcinoma of head and neck; PNI, perineural invasion.

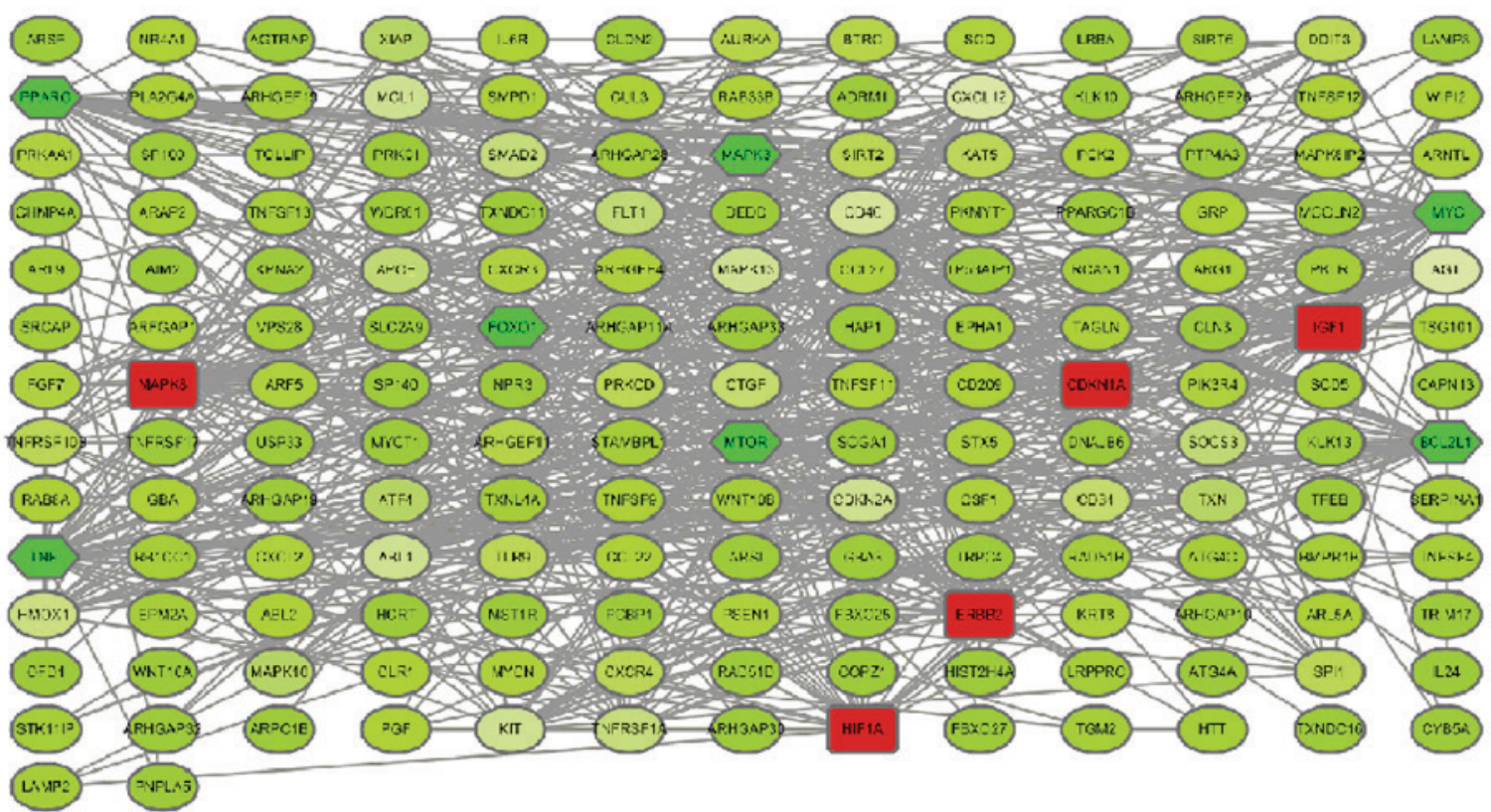

Figure 4. Based on the information of 239 autophagy-related genes contained in the STRING database, 171 nodes and 669 edges formed in the protein-protein interaction network. Red denotes upregulated genes, and green represents downregulated genes. 


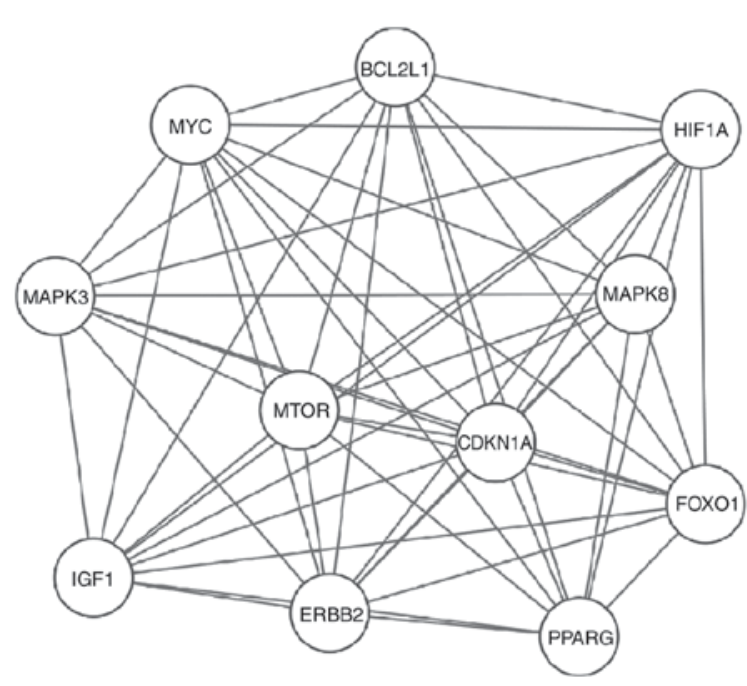

Figure 5. A sub-network of key proteins was constructed (degree $\geq 30$ ). The upregulated proteins include MAPK8, ERBB2, HIF1A, IGF1 and CDKN1A. The downregulated proteins include MYC, BCL2L1, mTOR, PPARG and FOXO1. MAPK8, mitogen-activated protein kinase 8; ERBB2, erb-b2 receptor tyrosine kinase 2 ; HIF, hypoxia-inducible factor; IGF, insulin growth factor; CDKN1A, cyclin dependent kinase inhibitor 1A; MYC, MYC proto-oncogene bHLH transcription factor; BCL2L1, B-cell lymphoma 2 like 1; mTOR, mammalian target of rapamycin; PPARG, peroxisome proliferator activated receptor $\gamma$; FOXO1, Forkhead box O1.

$\mathrm{r}=0.655$; Fig. 2C), but negatively associated with mTOR $(\mathrm{P}=0.002, \mathrm{r}=-0.748$; Fig. 2D) and BCL2L1 ( $\mathrm{P}=0.015, \mathrm{r}=-0.528$; Fig. 2E). Hierarchical clustering analysis revealed consistent results (Fig. 3).

PPI network and module screening. Based on the information of the 239 autophagy-related genes contained in the STRING database, 171 nodes and 669 edges formed (Fig. 4). A total of 11 nodes were regarded as key proteins (degree $\geq 30$ ) in the PPI network. This included upregulated genes, such as MAPK8 with highest node degree 41, followed by erb-b2 receptor tyrosine kinase 2 (ERBB2) (31), and HIF1A (30), and downregulated genes, such as tumor necrosis factor (TNF) with highest node degree 44, followed by MYC(42), BCL2L1 (36), mTOR (34), and peroxisome proliferator activated receptor $\gamma$ (PPAR $\gamma)$ (32). Modules of PPI network were screened and subnetworks were constructed (Fig. 5). In addition, a RAB23-related network predicted that there was a close association among RAB23, UBC (ubiquitin C) and ARFGAP1 based on post-translational regulation, suggesting a potential regulatory role of RAB23 in ARFGAP1 (Fig. 6).

\section{Discussion}

Cutaneous squamous cell carcinoma (cSCC) accounts for the second most common non-melanoma deriving skin cancer worldwide. Although most of cases can be successfully treated with surgery, there is a subset of lesions with invasion and metastasis, resulting in severe morbidity and mortality $(1,2,33)$. Histopathologically, lesions with thickness $>2 \mathrm{~mm}$, poor differentiation, invasion of the subcutaneous tissue or structures like vascular and lymphatic, or involvement of nerves $(>0.1 \mathrm{~mm}$ in diameter) predict poor prognosis in patients with cSCC $(22,34,35)$.
To date, although many gene expression data of SCCHN or cSCC have been analyzed by bioinformatics methods, few reports regarding the role of autophagy-related genes in cSCC have been recorded $(23,36,37)$.

In view of the variability of cSCC biological behavior, early identification of high-risk factors for PNI in CSCC is very important. However, to date, there has not been a gene signature, especially for autophagy-related genes, that can efficiently evaluate the degree of PNI in order to provide improved early intervention treatment.

In the present study, gene expression profile data were downloaded from the GEO database and analyzed for DEGs in cSCCHN using bioinformatics analysis, focusing on autophagy-related genes. A total of 2,858 DEGs were identified between cSCCHN with clinical PNI and cSCCHN samples, containing 921 upregulated and 1,937 downregulated genes. Of these, 239 autophagy-related DEGs (157 upregulated and 82 downregulated) were screened and clustered, employing series matrix files.

Increasing evidence has demonstrated that co-expressed genes, with similar expression profiles, frequently participate in parallel biological process. To better understand the interactions among the autophagy-related DEGs, GO and KEGG pathway analysis were performed. The GO term analysis indicated that upregulated DEGs were mainly involved in TNF-mediated signaling pathway, autophagy, positive regulation of transcription or MAP kinase activity and ERK1/2 cascade. Downregulated DEGs were involved in autophagy, signal transduction, glycosphingolipid metabolic process, macroautophagy and positive regulation of gene expression. This is consistent with the knowledge that defective function of TNF-mediated signaling pathway and autophagy is partly responsible for tumor progression and invasion.

Furthermore, by KEGG pathway analysis, the upregulated autophagy-related DEGs were enriched in cytokine-cytokine receptor interaction, pathways in cancer, ErbB signaling pathway, HIF-1 signaling pathway and transcriptional misregulation in cancer. The enriched KEGG pathways of the downregulated DEGs consisted of insulin resistance, pathways in cancer, FoxO signaling pathway and insulin signaling pathway. Previous studies have demonstrated that autophagy-related up and downregulated genes in human breast cancer can predict the survival rate and prognosis of breast cancer patients (38). Therefore, the key autophagy-related DEGs identified in the present study, namely HIF1A, MAPK8, mTOR, BCL2L1 and RAB23, may be involved in cSCCHN with clinical PNI. Based on the PPI network, the top degree key proteins were obtained. Among the DEGs, HIF1A and ERBB2 can be activated by corresponding ligands or external stimuli signals, further triggering signal transduction by downstream core member mTOR, or pathways such as IKKB-mTOR, PI3K-AKT-mTOR or MEK-ERK1/2-mTOR, and finally downregulate mTOR. Autophagy has the potential to promote either cell survival or cell death, depending on the context of different cancers, clinicopathological stages and post-irradiation status (39). Notably, mTOR is the first key autophagy-related gene and repressed mTOR may activate the autophagy process (40). At present, mTOR inhibitors, such as everolimus or temsirolimus, inhibit cell proliferation of head and neck cancer cell lines in vitro; however, in some phase I/II 


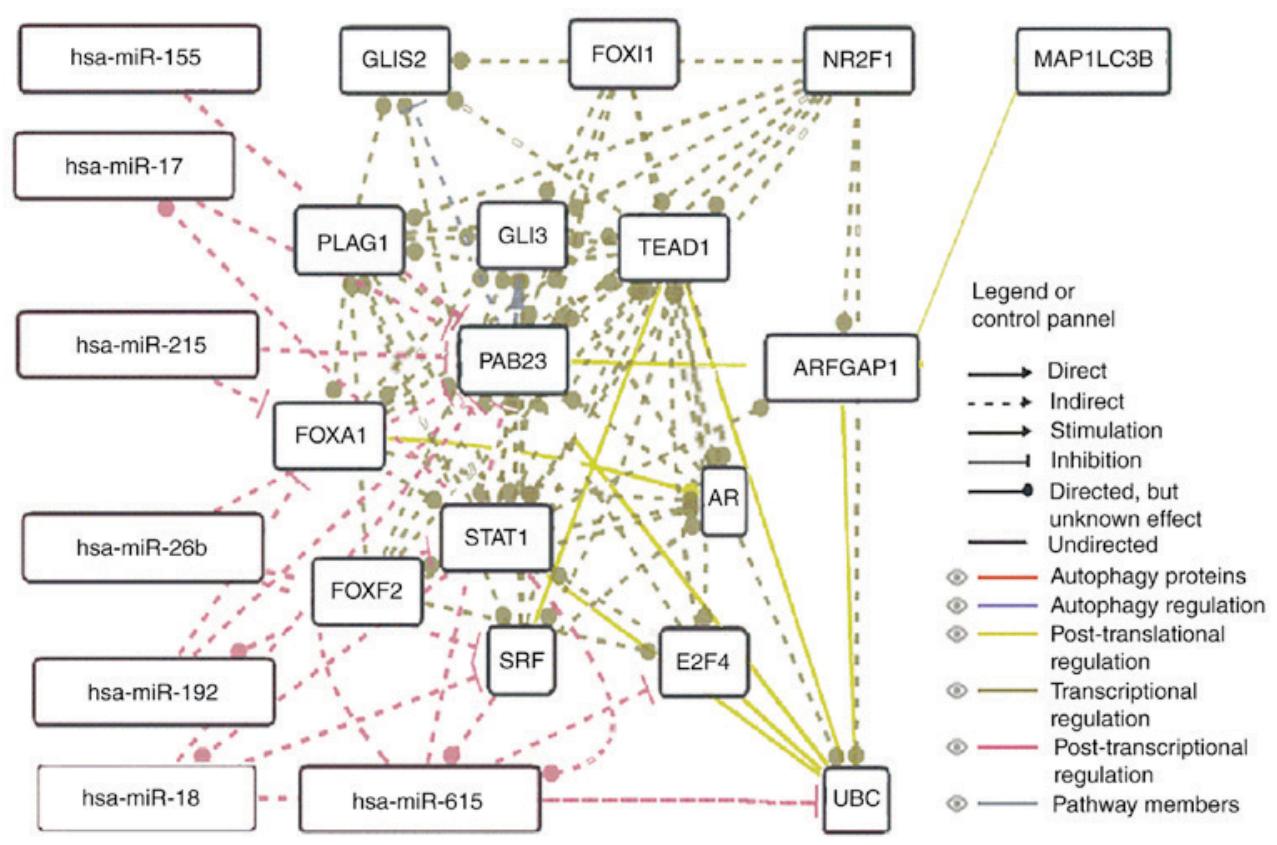

Figure 6. A RAB23 network was constructed denoting post-translational and transcriptional regulation and potential effectors in autophagy.

studies, only a partial response was observed and the curative effect was variable. Therefore, targeting both PI3K and mTOR concurrently is hypothesized to be more efficient. Two studies have demonstrated that NVP-BEZ235 (both a PI3K and mTOR kinase inhibitor) can induce autophagy in cells, and combination treatment with autophagy inhibitors, NVP-BEZ235 and radiation increased cell death $(41,42)$. The autophagy-related genes MAPK8, ERBB2 and HIF1A were found upregulated in tumor cells suggesting a constitutive activation of the autophagy machinery in cSCCHN with clinical PIN. Although TNF was identified as one of the key genes which exhibit the highest degree of connectivity, it was not included in the PPI subnetwork. Other post-transcription regulation mechanisms may therefore be involved, and this will need to be further verified in future studies.

The second key gene HIF1A, functions to buffer stressful response to hypoxia by activating transcription of multiple genes and to maintain cellular and systemic homeostasis. Therefore, HIF1A serves a key role in embryonic vascularization, angiogenesis in tumor and pathophysiology process of ischemic diseases. Hypoxia can activate autophagy in cancer cells and further induce clearance of p62 protein, suggesting a role for p62 in the regulation of cell survival responses $(43,44)$. In the oral squamous cell carcinoma cell line Tca8113, the HIF1A inhibitor PX-478 downregulated the expression levels of LC3-II/I and inhibited the autophagy process (45). Low oxygen levels in SCCHN promote more invasive phenotypes (46) and correlate with poor local control after radiation. Blocking EGFR, and hence PI3K-AKT signaling, led to improved oxygenation. Constitutively active AKT favors HIF-1-dependent gene transcription, which modulates angiogenesis, $\mathrm{pH}$, and glucose metabolism. In addition, other studies have highlighted the role of transcription factors p53, E2F and HIF1A in regulating the transcription of autophagy-related genes, including autophagy-related 5 (ATG5), BCL2-interacting protein 3 (BNIP3), Unc-51 like autophagy activating kinase 1 (ULK1), LC3 and DNA damage-regulated autophagy modulator (DRAM), subsequently activating autophagy in cancer cells in response to hypoxia or chemotherapy $(47,48)$. Furthermore, HIF1A appears to regulate the expression of MYC in a direct way and is regarded as the key node in the interactive network.

The third key gene MAPK8, also known as c-Jun $\mathrm{N}$-terminal kinase (JNK), participates in a wide variety of biological processes, such as proliferation, differentiation and transcription regulation. Various cell stimuli can activate MAPK8, which subsequently targets specific transcription factors, and finally mediates immediate-early gene expression. In addition, MAPK8 is involved in apoptosis induced by UV radiation, which is considered to be associated with the signal pathway of cytochrome c-mediated cell death. Furthermore, MAPK8 pathway is indispensable for TNF superfamily 10 (TNFSF)-induced autophagy. Blocking MAPK8 with the inhibitor SP600125 effectively reduced degradation of BCL2L1 and expression of the autophagy-suppressing BCL2L1-BECN1complex. Knockdown of TNF receptor associated factor 2 (TRAF2) or receptor interacting serine/threonine kinase 1 (RIPK1) by small interfering RNA effectively repressed TNFSF10-induced MAPK8 activation and autophagy levels (49).

RAB-like 3 (RAB13), as a member of the Rab subfamily of small GTPases, participates in controlling cell proliferation and vesicular trafficking. Recently, Zhang et al (50) demonstrated that knockdown of RABl3 in lung cancer cells significantly increased cell death with autophagy induction, as demonstrated by an elevated level of LC3-II. Interestingly, RABl3 knockdown was also associated with enhanced activation of MAPK8/9/10, except for MAPK11/12/13/14. Treatment withSP600125 (a MAPK8/9/10-specific inhibitor) significantly abolished RAB13 knockdown-induced LC3-II levels and autophagic cell death (50). Similar to RAB13, RAB23 is likely involved in the autophagy process in $\mathrm{CSCCHN}$ with clinical 
PNI: Nozawa et al (51) reported that RAB9A and RAB23 were novel partners of autophagy regulation against group A streptococcus infection. However, the autophagosome-like structure or autophagosome lacked direct evidence and needs to be additionally confirmed. Following treatment with $30 \mathrm{~mJ} / \mathrm{cm}^{2}$ UVB, wild-type RAB23 promotes the expression of LC3-II and Beclin1, while knockdown of RAB23 decreases the levels of LC3-II and Beclin 1 (52). Consistent with this result, the results of fluorescence microscopy demonstrated that wild-type RAB23 largely increases the number of autophagosomes (52). In addition, RAB23 promotes cell proliferation, migration and invasion in human astrocytoma, possibly through upregulating Rac1 activity (53). The present data indicated ARFGAP1 may be an effector of RAB23, which is associated with the autophagic marker LC3B. Overall, RAB23 is likely to indirectly promote autophagy.

As previously discussed, the anti-apoptotic proteins of the BCL-2 family negatively regulate the assembly of phagophore membrane. BCL-2 and BCL2L1 were proved to inhibit autophagy by binding Beclin 1 at the $\mathrm{BH} 3$ domain site $(54,55)$. BCL2L1 protein is located at the outer mitochondrial membrane and functions to regulate the opening of the outer mitochondrial membrane channel, a kind of voltage-dependent anion channel (VDAC). VDAC further regulates the mitochondrial membrane potential and controls the production of reactive oxygen species (ROS) and release of cytochrome c, both of which effectively induce cell apoptosis (56). Knocking down myeloid cell leukemia sequence-1 (Mcl-1) sensitizes oral SCC cells to ABT-737 (a BH3 mimetic), which binds to BCL2L1 but not Mcl-1 (57).

Dysregulation of autophagy contributes to the progression of cancer. Recently, based on gene expression profiles, $\mathrm{Gu}$ et al (38) reported the predictive value of autophagy for assessing prognosis of breast cancer. The authors identified a set of eight autophagy-related genes, including BCL2, GAPDH and vascular endothelial growth factor A, which were closely related with overall survival in breast cancer (38). Further analysis demonstrated that the prognostic value of the autophagy signature was independent of known clinical prognostic factors, such as ERBB2 status, lymph node status and p53 mutation status. The results from the present study demonstrated that a close correlation may also exist between autophagy and cSCC outcome, and that autophagy-related genes are promising treatment target for skin cancer.

In short, a total of 239 DEGs were identified between cSCCHN with clinical PNI and $\mathrm{cSCCHN}$ without neural involvement. Genes such as MAPK8, HIF1A, mTOR, BCL2L1 and RAB23 may be potential therapeutic target genes in cSCCHN. Furthermore, RAB23 may promote autophagy in cSCC through ARFGAP1 in an indirect way.

As far as clinical significance of these target genes is concerned, several pathway inhibitors have exhibited antitumor activity in vitro or in preclinical models, but this has not always provided meaningful benefits to patients with SCCHN. Therefore, agents or drugs that target multiple receptors alone or in combination with other medicines will likely provide the most efficient therapeutic effect for patients.

The present study has several limitations, such as small sample size and lack of experimental verification, possibly generating false positive results. Therefore, further experimental studies with larger sample sizes will be needed in the future to confirm the results.

\section{Acknowledgements}

The present study was funded by the National Natural Sciences Foundation of China (grant nos. 31371412, 81572680 and 81673043).

\section{Competing interests}

The authors declare that they have no competing interests.

\section{References}

1. Rogers HW, Weinstock MA, Harris AR, Hinckley MR, Feldman SR, Fleischer AB, Fleischer AB and Coldiron BM: Incidence estimate of non-melanoma skin cancer in the United States, 2006. Arch Dermatol 146: 283-287, 2010.

2. Eisemann N, Waldmann A, Geller AC, Weinstock MA, Volkmer B, Greinert R, Breitbart EW and Katalinic A: Non-melanoma skin cancer incidence and impact of skin cancer screening on incidence. J Invest Dermatol 134: 43-50, 2014.

3. Narayanan DL, Saladi RN and Fox JL: Ultraviolet radiation and skin cancer. Int J Dermatol 49: 978-986, 2010.

4. Veness MJ: Defining patients with high-risk cutaneous squamous cell carcinoma. Australas J Dermatol 47: 28-33, 2006.

5. Echarri MJ, Lopez-Martin A and Hitt R: Targeted therapy in locally advanced and Recurrent/metastatic head and neck squamous cell carcinoma (LA-R/M HNSCC). Cancers (Basel) 8: E27, 2016.

6. Yoshihara N, Takagi A, Ueno T and Ikeda S: Inverse correlation between microtubule-associated protein 1A/1B-light chain 3 and p62/sequestosome-1 expression in the progression of cutaneous squamous cell carcinoma. J Dermatol 41: 311-315, 2014.

7. Okura R and Nakamura M: Overexpression of autophagy-related beclin-1 in cutaneous squamous cell carcinoma with lymph-node metastasis. Eur J Dermatol 21: 1002-1003, 2011.

8. Klionsky DJ: Autophagy: From phenomenology to molecular understanding in less than a decade. Nat Rev Mol Cell Biol 8: 931-937, 2007.

9. Rabinowitz JD and White E: Autophagy and metabolism. Science 330: 1344-1348, 2010.

10. Levine B and Klionsky DJ: Development by self-digestion: Molecular mechanisms and biological functions of autophagy. Dev Cell 6: 463-477, 2004.

11. Jin S and White E: Role of autophagy in cancer: Management of metabolic stress. Autophagy 3: 28-31, 2007.

12. Mathew R, Karantza-Wadsworth V and White E: Role of autophagy in cancer. Nat Rev Cancer 7: 961-967, 2007.

13. Lorin S, Hamai A, Mehrpour M and Codogno P: Autophagy regulation and its role in cancer. Semin Cancer Biol 23: 361-379, 2013.

14. Liu JL, Chen FF, Lung J, Lo CH, Lee FH, Lu YC and Hung CH: Prognostic significance of p62/SQSTM1 subcellular localization and LC3B in oral squamous cell carcinoma. Br J Cancer 111: 944-954, 2014.

15. Claerhout S, Verschooten L, Van Kelst S, De Vos R, Proby C, Agostinis P and Garmyn M: Concomitant inhibition of AKT and autophagy is required for efficient cisplatin-induced apoptosis of metastatic skin carcinoma. Int J Cancer 127: 2790-2803, 2010.

16. Wright TJ, McKee C, Birch-Machin MA, Ellis R, Armstrong JL and Lovat PE: Increasing the therapeutic efficacy of docetaxel for cutaneous squamous cell carcinoma through the combined inhibition of phosphatidylinositol 3-kinase/AKT signalling and autophagy. Clin Exp Dermatol 38: 421-423, 2013.

17. Fan TF, Bu LL, Wang WM, Ma SR, Liu JF, Deng WW, Mao L, Yu GT, Huang CF, Liu B, et al: Tumor growth suppression by inhibiting both autophagy and STAT3 signaling in HNSCC. Oncotarget 6: 43581-43593, 2015.

18. Barrette K, Van Kelst S, Wouters J, Marasigan V, Fieuws S, Agostinis P, van den Oord J and Garmyn M: Epithelial-mesenchymal transition during invasion of cutaneous squamous cell carcinoma is paralleled by AKT activation. Br J Dermatol 171: 1014-1021, 2014. 
19. Choi SR, Chung BY, Kim SW, Kim CD, Yun WJ, Lee MW, Choi JH and Chang SE: Activation of autophagic pathways is related to growth inhibition and senescence in cutaneous squamous cell carcinoma. Exp Dermatol 23: 718-724, 2014.

20. Balamucki CJ, Mancuso AA, Amdur RJ, Kirwan JM, Morris CG, Flowers FP, Stoer CB, Cognetta AB and Mendenhall WM: Skin carcinoma of the head and neck with perineural invasion. Am J Otolaryngol 33: 447-454, 2012.

21. Warren TA, Panizza B, Porceddu SV, Gandhi M, Patel P, Wood M, Nagle CM and Redmond M: Outcomes after surgery and postoperative radiotherapy for perineural spread of head and neck cutaneous squamous cell carcinoma. Head Neck 38: 824-831, 2016.

22. Warren TA, Broit N, Simmons JL, Pierce CJ, Chawla S, Lambie DL, Quagliotto G, Brown IS, Parsons PG, Panizza BJ and Boyle GM: Expression profiling of cutaneous squamous cell carcinoma with perineural invasion implicates the p53 pathway in the process. Sci Rep 6: 34081, 2016.

23. Padilla RS, Sebastian S, Jiang Z, Nindl I and Larson R: Gene expression patterns of normal human skin, actinic keratosis, and squamous cell carcinoma: A spectrum of disease progression. Arch Dermatol 146: 288-293, 2010.

24. Ra SH,Li X and Binder S: Molecular discrimination of cutaneous squamous cell carcinoma from actinic keratosis and normal skin. Mod Pathol 24: 963-973, 2011.

25. Mitsui H, Suárez-Farinas M, Gulati N, Shah KR, Cannizzaro MV, Coats I, Felsen D, Krueger JG and Carucci JA: Gene expression profiling of the leading edge of cutaneous squamous cell carcinoma: IL-24-driven MMP-7. J Invest Dermatol 134: 1418-1427, 2014.

26. Farshchian M, Nissinen L, Siljamäki E, Riihilä P, Toriseva M, Kivisaari A, Ala-Aho R, Kallajoki M, Veräjänkorva E, Honkanen HK, et al: EphB2 promotes progression of cutaneous squamous cell carcinoma. J Invest Dermatol 135: 1882-1892, 2015.

27. Jian Q, Miao Y, Tang L, Huang M, Yang Y, Ba W, Liu Y, Chi S and $\mathrm{Li}$ : Rab23 promotes squamous cell carcinoma cell migration and invasion via integrin $\beta 1 /$ Racl pathway. Oncotarget 7 : $5342-5352,2016$

28. Turei D, Földvari-Nagy L, Fazekas D, Módos D, Kubisch J Kadlecsik T, Demeter A, Lenti K, Csermely P, Vellai T and Korcsmáros T: Autophagy regulatory network-a systems-level bioinformatics resource for studying the mechanism and regulation of autophagy. Autophagy 11: 155-165, 2015

29. Huang da W, Sherman BT and Lempicki RA: Systematic and integrative analysis of large gene lists using DAVID bioinformatics resources. Nat Protoc 4: 44-57, 2009.

30. de Hoon MJ, Imoto S, Nolan J and Miyano S: Open source clustering software. Bioinformatics 20: 1453-1454, 2004.

31. Saldanha AJ: Java Treeview-extensible visualization of microarray data. Bioinformatics 20: 3246-3248, 2004.

32. Szklarczyk D, Morris JH, Cook H, Kuhn M, Wyder S, Simonovic M, Santos A, Doncheva NT, Roth A, Bork P, et al: The STRING database in 2017: Quality-controlled protein-protein association networks, made broadly accessible. Nucleic Acids Res 45: D362-D368, 2017.

33. Parikh SA, Patel VA and Ratner D: Advances in the management of cutaneous squamous cell carcinoma. F1000Prime Rep 6: 70, 2014.

34. Miller SJ: Defining, treating, and studying very high-risk cutaneous squamous cell carcinomas. Arch Dermatol 146: $1292-1295,2010$.

35. Ross AS, Whalen FM, Elenitsas R, Xu X, Troxel AB and Schmults CD: Diameter of involved nerves predicts outcomes in cutaneous squamous cell carcinoma with perineural invasion: an investigator-blinded retrospective cohort study. Dermatol Surg 35: 1859-1866, 2009.

36. Nindl I, Dang C, Forschner T, Kuban RJ, Meyer T, Sterry W and Stockfleth E: Identification of differentially expressed genes in cutaneous squamous cell carcinoma by microarray expression profiling. Mol Cancer 5: 30, 2006.

37. Rothenberg SM and Ellisen LW: The molecular pathogenesis of head and neck squamous cell carcinoma. J Clin Invest 122 : 1951-1957, 2012

38. Gu Y, Li P, Peng F, Zhang M, Zhang Y, Liang H, Zhao W, Qi L, Wang H, Wang C and Guo Z: Autophagy-related prognostic signature for breast cancer. Mol Carcinog 55: 292-299, 2016.
39. Kim KW, Mutter RW, Cao C, Albert JM, Freeman M, Hallahan DE and Lu B: Autophagy for cancer therapy through inhibition of pro-apoptotic proteins and mammalian target of rapamycin signaling. J Biol Chem 281: 36883-36890, 2006.

40. OFarrellF,Rusten TEand StenmarkH:Phosphoinositide3-kinases as accelerators and brakes of autophagy. FEBS J 280: 6322-6337, 2013.

41. Cerniglia GJ, Karar J, Tyagi S, Christofidou-Solomidou M, Rengan R, Koumenis C and Maity A: Inhibition of autophagy as a strategy to augment radiosensitization by the dual phosphatidylinositol 3-kinase/mammalian target of rapamycin inhibitor NVP-BEZ235. Mol Pharmacol 82: 1230-1240, 2012.

42. Kim KW, Myers CJ, Jung DK and Lu B: NVP-BEZ-235 enhances radiosensitization via blockade of the $\mathrm{PI} 3 \mathrm{~K} / \mathrm{mTOR}$ pathway in cisplatin-resistant non-small cell lung carcinoma. Genes Cancer 5: 293-302, 2014.

43. Pursiheimo JP, Rantanen K, Heikkinen PT, Johansen T and Jaakkola PM: Hypoxia-activated autophagy accelerates degradation of SQSTM1/p62. Oncogene 28: 334-344, 2009.

44. Li X and Fan Z: The epidermal growth factor receptor antibody cetuximab induces autophagy in cancer cells by downregulating HIF-1alpha and Bcl-2 and activating the beclin 1/hVps34 complex. Cancer Res 70: 5942-5952, 2010.

45. Li YN, Hu JA and Wang HM: Inhibition of HIF-1 $\alpha$ affects autophagy mediated glycosylation in oral squamous cell carcinoma cells. Dis Markers 2015: 239479, 2015.

46. Harris AL: Hypoxia - a key regulatory factor in tumour growth. Nat Rev Cancer 2: 38-47, 2002.

47. Pouysségur J, Dayan F and Mazure NM: Hypoxia signalling in cancer and approaches to enforce tumour regression. Nature 441: 437-443, 2006.

48. Bohensky J, Shapiro IM, Leshinsky S, Terkhorn SP, Adams CS and Srinivas V: HIF-1 regulation of chondrocyte apoptosis: Induction of the autophagic pathway. Autophagy 3: 207-214, 2007.

49. He W, Wang Q, Xu J, Xu X, Padilla MT, Ren G, Gou X and Lin Y: Attenuation of TNFSF10/TRAIL-induced apoptosis by an autophagic survival pathway involving TRAF2- and RIPK1/RIP1-mediated MAPK8/JNK activation. Autophagy 8 : 1811-1821, 2012.

50. Zhang W, Sun J and Luo J: High expression of Rab-like 3 (Rabl3) is associated with poor survival of patients with non-small cell lung cancer via repression of MAPK8/9/10-mediated autophagy. Med Sci Monit 22: 1582-1588, 2016.

51. Nozawa T, Aikawa C, Goda A, Maruyama F, Hamada S and Nakagawa I: The small GTPases Rab9A and Rab23 function at distinct steps in autophagy during Group A Streptococcus infection. Cell Microbiol 14: 1149-1165, 2012.

52. Zheng LQ, Chi SM and Li CX: Rab23's genetic structure, function and related diseases: A review. Biosci Rep 37: BSR20160410, 2017.

53. Wang M, Dong Q and Wang Y: Rab23 is overexpressed in human astrocytoma and promotes cell migration and invasion through regulation of Rac1. Tumour Biol 37: 11049-11055, 2016.

54. Maiuri MC, Le Toumelin G, Criollo A, Rain JC, Gautier F, Juin P, Tasdemir E, Pierron G, Troulinaki K, Tavernarakis N, et al: Functional and physical interaction between $\mathrm{Bcl}-\mathrm{X}(\mathrm{L})$ and a BH3-like domain in Beclin-1. EMBO J 26: 2527-2539, 2007.

55. Erlich S, Mizrachy L, Segev O, Lindenboim L, Zmira O, Adi-Harel S, Hirsch JA, Stein R and Pinkas-Kramarski R: Differential interactions between Beclin 1 and Bcl-2 family members. Autophagy 3: 561-368, 2007.

56. Yuan J, Zhang Y, Sheng Y, Fu X, Cheng H and Zhou R: MYBL2 guides autophagy suppressor VDAC2 in the developing ovary to inhibit autophagy through a complex of VDAC2-BECN1-BCL2L1 in mammals. Autophagy 11: 1081-1098, 2015.

57. Maji S, Samal SK, Pattanaik L, Panda S, Quinn BA, Das SK, Sarkar D, Pellecchia M, Fisher PB and Dash R: Mcl-1 is an important therapeutic target for oral squamous cell carcinomas. Oncotarget 6: 16623-16637, 2015.

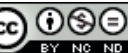

This work is licensed under a Creative Commons Attribution-NonCommercial-NoDerivatives 4.0 International (CC BY-NC-ND 4.0) License. 